\title{
Multivariable analysis of factors associated with USMLE scores across U.S. medical schools
}

\author{
Arash Ghaffari-Rafi ${ }^{1,2^{*}} \mathbb{D}$, Rachel Elizabeth Lee ${ }^{1 \dagger}$, Rui Fang ${ }^{1}$ and J. Douglas Miles ${ }^{1}$
}

\begin{abstract}
Background: Gauging medical education quality has always remained challenging. Many studies have examined predictors of standardized exam performance; however, data sets do not distinguish by institution or curriculum. Our objective is to present a summary of variables associated with the United States Medical Licensing Examination (USMLE) scores, and thus identify institutions (and therefore curriculums) which deviate from trend lines by producing higher USMLE scores despite having lower entrance grade point averages and medical college admissions test (MCAT) scores.

Methods: Data was obtained from U.S. News and World Report's 2014 evaluation of allopathic U.S. medical schools. A univariate analysis was performed first for each variable using two sample t-test or Wilcoxon rank sum test for categorical variables, and Pearson or Spearman correlation coefficients for continuous variables. A multivariable linear regression model was developed to identify the factors contributing to USMLE scores. All statistical analyses were two-sided and performed using SAS software version 9.4 (SAS Institute Inc., Cary, NC).

Results: Univariate analysis reveals a significant association between USMLE Step 1 and 2 scores with medical college admissions test scores, grade point averages, school type (private vs. public), full-time faculty-to-student ratio, National Institute of Health funds, residency director assessment score, peer assessment score, and class size. Of these nine variables, MCAT scores and Step 1 scores display the strongest correlation (corr $=0.72, P<.0001$ ). Multivariable analysis also supports a significant association between MCAT scores and Step scores, meanwhile National Institute of Health funding size demonstrates a negative correlation with USMLE Step 2 scores. Although MCAT scores and National Institute of Health funds are significantly associated with USMLE performance, six outlier institutions were identified, producing higher USMLE scores than trend line predictions.
\end{abstract}

Conclusions: Outlier institutions produce USMLE scores that do not follow expected trend lines. Their performance might be explainable by differences in curriculum. Having identified these institutions, their curriculums can be further studied to determine what factors enhance student learning.

Keywords: United States medical licensing examination, USMLE, Evaluation, Student learning, Curriculum, Assessment

\footnotetext{
* Correspondence: arash.ghaffari-rafi.18@ucl.ac.uk

Arash Ghaffari Rafi and Rachel E. Lee are Co-first authors.

'University of Hawai'i at Mānoa John A. Burns School of Medicine, Honolulu,

Hawai'i, USA

${ }^{2}$ Queen Square Institute of Neurology, University College London, London, UK
}

(c) The Author(s). 2019 Open Access This article is distributed under the terms of the Creative Commons Attribution 4.0 International License (http://creativecommons.org/licenses/by/4.0/), which permits unrestricted use, distribution, and reproduction in any medium, provided you give appropriate credit to the original author(s) and the source, provide a link to the Creative Commons license, and indicate if changes were made. The Creative Commons Public Domain Dedication waiver (http://creativecommons.org/publicdomain/zero/1.0/) applies to the data made available in this article, unless otherwise stated. 


\section{Background}

Gauging medical education quality has always remained challenging due to the myriad of factors that can be assessed, including those which are difficulty to quantify-such as adherence to the medical school's mission statement. Despite such challenges, prior medical school assessments have emphasized school admissions rate, entering class Medical College Admissions Test (MCAT) and grade point averages (GPA), full-time faculty-to-student ratio, and National Institute of Health (NIH) funding [1-3]. Meanwhile, two forms of student evaluation that occur during the time of medical studies include assessments in clinical clerkships and United States Medical Licensing Examination (USMLE) exams; due to variability in scoring systems for clinical clerkships, the most consistent measurement of school product is the USMLE Step exams [4]. Step 1 assesses basic science knowledge, whereas Step 2 focuses on clinical understanding [4]. These exams are the primary academic criteria for residency selection, for to an extent they provide a gauge of student learning $[5,6]$.

Many studies have examined predictors of standardized exam performance; however, data sets do not distinguish by institution or curriculum (i.e., problem based learning, lectures, team based learning, etc.). Moderate correlations have been identified between USMLE Step 1, MCAT, and undergraduate GPA [7-11]. Performance on Step 2 Clinical Knowledge (CK) exam has also been associated with performance on USMLE Step 1 and the MCAT [12-15]. However, numerous predictors of USMLE performance, including subjective predictors (i.e. peer assessment score), have not been compared against objective predictors (i.e. standardized exam scores), and thus, their reliability is unknown. This study examines multiple variables to determine which factors play a greater role in determining medical student success, as well as identifies institutions that significantly deviate from expected trend lines, and thus identify those curricula that may potentially excel in efficiently educating students.

\section{Methods}

\section{Design and setting}

Data was collected from a publicly accessible database, U.S. News and World Report's (USN\&WR), and does not contain specific student identifiers. Institutional review board exemption for waivers of informed consent was attained from the University of Hawai'i at Mānoa, Office of Research Compliance. Permission to utilize data from USN\&WR in a non-commercial manner was attained from the Permissions Office and the Director of Specialty Marketing at USN\&WR. Only publicly available data was utilized in our analysis.

USN\&WR (https://www.usnews.com/best-graduate-scho ols/top-medical-schools/research-rankings) surveyed 130 medical schools fully accredited by the Liaison Committee on Medical Education. Of those schools, 100 provided data. 2014 data was compiled to compare average USMLE Step 1 and Step 2 scores against nine variables: median undergraduate GPA, median MCAT, school type (private vs public), full-time faculty-to-student ratio, NIH funds granted to the medical school and affiliated hospitals, NIH research grant funds per faculty member, peer assessment score, residency directors assessment, and total medical school enrollment.

Median MCAT total scores and undergraduate GPAs were obtained from students taking USMLE in 2014. Faculty resources were measured as the ratio of full-time science and full-time clinical faculty to full-time M.D. students. Research activity was based on the total dollar amount of grants awarded by the NIH to the medical school and its affiliated hospitals, and of NIH grant funding per full-time faculty member.

The peer assessment score was based on subjective ratings collected from medical school deans, deans of academic affairs, department heads of internal medicine, and directors of admissions from other medical schools. These respondents rated programs on a scale from 1 (marginal) to 5 (outstanding). For fair evaluation, individuals with limited knowledge about a medical school were requested to select the neutral response "don't know," from the scale of response options. A school's average score was the average rating of all the respondents who rated it. Residency program directors were also asked to rate programs using the same 5-point scale. Each medical school reported total medical school enrollment in year 2014 to USN\&WR.

\section{Statistical analysis}

The data was summarized by descriptive statistics: mean with standard deviation (SD) or median with minimum and maximum for continuous variables (based on distribution) such as Step scores, and frequency and percentage for categorical variables such as school type (public or private). To access the association with Step scores, a univariate analysis was performed first for each variable using two sample t-test or Wilcoxon rank sum test for categorical variables, and Pearson or Spearman correlation coefficients for continuous variables. A multivariable linear regression model was developed to identify the factors contributing to USMLE scores. Significant variables in the univariate analysis were considered to be included into the model. All statistical analyses were two-sided and performed using SAS software version 9.4 (SAS Institute Inc., Cary, NC). An alpha level of 0.05 was used to determine statistical significance.

\section{Results}

100 U.S. medical schools reported both USMLE Step 1 and 2 scores, and thus are the focus of this analysis. 
Average Step 1 and 2 scores are $230.5(\mathrm{SD}=6.0)$ and $240.0(\mathrm{SD}=4.9)$, respectively. Factors that associate with USMLE scores are summarized in Table 1. Fifty-nine (59.0\%) of schools are public. On average, the median GPA and MCAT scores are $3.7(\mathrm{SD}=0.09)$ and 32.1 (SD $=2.6$ ), respectively. The median full-time faculty-student ratio is 1.8 (ranged from 0.2 to 14.9). The median $\mathrm{NIH}$ funds granted to the medical school and affiliated hospitals are 88.9 million (ranged from 1.8 to 1412.9 million). The median $\mathrm{NIH}$ research funds per faculty member are 87.47 thousand (ranged from 4.57 to 381.84 thousand). On average, the residency directors' assessment score is 3.4 $(\mathrm{SD}=0.6)$ and the peer assessment score is 3.1 (SD $=0.7$ ). The median of total medical school enrollment in the year 2014 is 631.5 (ranged from 216 to 1377).

The association between USMLE scores and potential factors are summarized in Table 2. There are statistically significant correlations between average Step 1 score and median GPA (corr $=0.55, P<.0001$ ), median MCAT total score (corr $=0.72, P<.0001$ ), full-time faculty-tostudent ratio (corr $=0.47, P<.0001)$, NIH funds granted to medical schools and affiliated hospitals (corr $=0.58, P$ $<.0001)$, NIH research grant funds per faculty member (corr $=0.54, P<.0001$ ), residency directors assessment score (corr $=0.60, P<.0001$ ), and peer assessment score (corr $=0.62, P<.0001$ ). There is a significant difference between private and public schools in Step 1 scores $(P<.0001)$. On average, private schools have around a five-point higher average Step 1 score, compared to public schools (233.2 vs. 228.6). Regarding average Step 2 scores, there are statistically significant correlations with Step 1 score (corr $=0.54, P<.0001$ ), median GPA $\quad($ corr $=0.49, \quad P<.0001)$, median MCAT total

Table 1 Summary of USMLE scores and potential factors

\begin{tabular}{ll}
\hline Variable & \\
\hline Avg. STEP1 score, mean (SD) & $230.5(6.0)$ \\
Avg. STEP2 score, mean (SD) & $240.0(4.9)$ \\
School Type, n (\%) & \\
$\quad$ Private & $41(41.0 \%)$ \\
$\quad$ Public & $59(59.0 \%)$ \\
Median GPA, mean (SD) & $3.7(0.09)$ \\
Median MCAT total score, mean (SD) & $32.1(2.6)$ \\
Full-Time Faculty-Student Ratio, median (min, max) & $1.8(0.2,14.9)$ \\
NIH Funds Granted to Medical School and Affiliated & $88.9(1.8,1412.9)$ \\
Hospitals in millions, median (min, max) & \\
NIH Research Grant Funds Per Faculty Member in & $87.47(4.57,381.84)$ \\
thousands, median (min, max) & \\
Residency Directors Assessment Score out of 5, & $3.4(0.6)$ \\
mean (SD) & \\
Peer Assessment Score out of 5, mean (SD) & $3.1(0.7)$ \\
Total Medical School Enrollment, median (min, max) & $631.5(216,1377)$ \\
\hline
\end{tabular}

score (corr $=0.60, P<.0001$ ), full-time faculty-to-student ratio (corr $=0.35, P=0.0004$ ), NIH funds granted to medical schools and affiliated hospitals (corr $=0.46, P<.0001$ ), $\mathrm{NIH}$ research grant funds per faculty member (corr $=0.35$, $P=0.0005)$, residency directors assessment score (corr $=$ $0.47, P<.0001$ ), and peer assessment score (corr $=0.49, P$ $<.0001)$. Compared to public schools, private schools have a slightly higher Step 2 score (241.3 vs. $239.2, P=0.051$ ).

Variables with a significant bivariate relationship to Step 1 score were entered into a linear model to predict Step 1 score. These variables include: median GPA, median MCAT total score, school type, full-time faculty-tostudent ratio, NIH funds granted to medical schools and affiliated hospitals, NIH research grant funds per faculty member, residency director assessment score and peer assessment score. Results are presented in Table 3. The results of the regression indicate that eight variables explained $58.4 \%$ of the variance $\left(\mathrm{R}^{2}=0.584, P<.0001\right)$. Higher median MCAT significantly predicted higher Step 1 score $(\beta=1.28, P=0.0002)$.

Variables with a significant bivariate relationship to Step 2 score were entered into a linear model to predict Step 2 score. These variables include: average Step 1 score, median GPA, median MCAT total score, school type, full-time faculty-to-student ratio, $\mathrm{NIH}$ funds granted to medical schools and affiliated hospitals, NIH research grant funds per faculty member, residency director assessment score, and peer assessment score. Results are present in Table 4. The results of the regression indicate that nine variables explained $46.9 \%$ of the variance $\left(R^{2}=0.469, P\right.$ $<.0001)$. Change of the following variables significantly predicts higher Step 2 scores: higher median MCAT total score $(\beta=1.11, P=0.012)$ and lower $\mathrm{NIH}$ research grant funds per faculty member $(\beta=-$ $0.02, P=0.039$ ).

\section{Additional analysis to identify outlier and influential points (fit diagnostic)}

The studentized residual ( $r$ ) and leverage (lev) were assessed to identify the schools that are potential outliers or have potential influences on regression coefficients estimates. For multivariable linear model for Step 1 score, the potential outliers are University of MissouriColumbia School of Medicine $(r=2.944)$ and University of Arkansas $(r=-3.089)$; the potential influence points are Harvard University with the largest leverage value of 0.792, followed by Mayo Medical School (lev $=0.715)$, Morehouse School of Medicine $(\mathrm{lev}=0.378)$, University of Washington (lev $=0.229)$, New York University (lev = $0.222)$, and Stanford University $(\mathrm{lev}=0.208)$. For the multivariable linear model for Step 2 score, the potential outliers are Emory University $(r=2.662)$, University of North Carolina $(r=2.164)$, University of Missouri-Columbia 
Table 2 Results of Univariate analysis with USMLE scores

\begin{tabular}{lllll}
\hline Variable & Avg. STEP1 score & $P$-value & Avg. STEP2 score & $P$-value \\
\hline Avg. STEP1 score & NA & NA & 0.54 & 0.49 \\
Median GPA & 0.55 & $<.0001$ & $<.0001$ \\
Median MCAT total score & 0.72 & $<.0001$ & 0.60 & $<.0001$ \\
School type, mean (SD) & & $<.0001$ & $241.3(5.7)$ & 0.051 \\
$\quad$ Private & $233.2(6.3)$ & $239.2(4.1)$ & 0.35 \\
$\quad$ Public & $228.6(5.0)$ & 0.001 & 0.46 \\
Full-Time Faculty-Student Ratio & 0.47 & $<.0001$ & 0.35 & 0.0004 \\
NIH Funds Granted to Medical School and Affiliated Hospitals in millions & 0.58 & $<.0001$ & $<.0001$ & 0.47 \\
NIH Research Grant Funds Per Faculty Member in thousands & 0.54 & $<.0001$ & 0.0001 \\
Residency Directors Assessment Score out of 5 & 0.60 & $<.0001$ & 0.49 & 0.02 \\
Peer Assessment Score out of 5 & 0.62 & 0.33 & $<.0001$ \\
Total Medical School Enrollment & -0.10 & $<.0001$ \\
\hline
\end{tabular}

School of Medicine $(r=2.002)$, Uniformed Service University of the Health Sciences $(r=-2.345)$, and Duke University $(r=-2.646)$, and the potential influence points are Harvard University (lev $=0.793)$, Mayo Medical School $(\mathrm{lev}=0.715)$, Morehouse School of Medicine (lev $=0.381)$, University of Washington (lev $=0.231)$ and New York University $(\mathrm{lev}=0.223)$. In Figs. 1 and 2, the observations outside two horizontal lines are potential outliers and the observations beyond the vertical line are potential influences.

\section{Discussion}

2014 dataset collected by USN\&WR are comparable to publically available Association of American Medical Colleges (AAMC) data [16, 17]. The average AAMC Step 1 score of $229 \quad(\mathrm{SD}=20)$ is comparable to USN\&WR average of $230.5(\mathrm{SD}=6.0)$, AAMC median GPA of $3.69(\mathrm{SD}=0.25)$ is comparable to USN\&WR

Table 3 Results of multivariable linear regression model for STEP 1 score

\begin{tabular}{llll}
\hline Variable & $\begin{array}{l}\text { Parameter } \\
\text { Estimate }\end{array}$ & $\begin{array}{l}\text { Standard } \\
\text { Error }\end{array}$ & P-value \\
\hline Intercept & 153.58 & 21.83 & $<.0001$ \\
Median GPA & 10.01 & 6.84 & 0.15 \\
Median MCAT total score & 1.28 & 0.33 & 0.0002 \\
School type, Private vs. Public & 1.36 & 1.01 & 0.19 \\
Full-Time Faculty-Student Ratio & -0.17 & 0.30 & 0.57 \\
NIH Funds Granted to Medical & 0.0004 & 0.0042 & 0.93 \\
School and Affiliated Hospitals & & & 0.85 \\
in millions & & 0.01 & \\
NIH Research Grant Funds Per & 0.002 & & 0.18 \\
Faculty Member in thousands & & 2.77 & 0.20 \\
Residency Directors Assessment & -3.82 & & \\
Score out of 5 & & 3.61 &
\end{tabular}

GPA of 3.7 ( $\mathrm{SD}=0.09)$, and AAMC median MCAT score of 31.4 $(\mathrm{SD}=3.9)$ is comparable to USN\&WR MCAT of 32.1 ( $S D=2.6)$. Variables unique to USN\&WR data are residency director assessment score and peer assessment score.

Univariate analysis (Table 2) suggests that all measured variables except total medical student enrollment are significant predictors of Step 1 and Step 2 scores, with MCAT having the highest correlation. Such corresponds with other studies utilizing different data sets, which indicate that MCAT is a strong predictor of medical school success, and thus positively correlates with Step scores $[8,18]$. On the other hand, school type is a marginally significant predictor of Step 2 scores as compared to Step 1 scores. One possible explanation for the difference between public and private medical schools, is that public institutions attain significant state funding.

Table 4 Results of multivariable linear regression model for STEP 2 score

\begin{tabular}{llll}
\hline Variable & $\begin{array}{l}\text { Parameter } \\
\text { Estimate }\end{array}$ & $\begin{array}{l}\text { Standard } \\
\text { Error }\end{array}$ & P-value \\
\hline Intercept & 148.45 & 25.3 & $<.0001$ \\
Avg. STEP1 score & 0.13 & 0.10 & 0.20 \\
Median GPA & 7.30 & 6.33 & 0.26 \\
Median MCAT total score & 1.10 & 0.33 & 0.0012 \\
School type, Private vs. Public & -0.99 & 0.94 & 0.30 \\
Full-Time Faculty-Student Ratio & -0.147 & 0.27 & 0.87 \\
NIH Funds Granted to Medical & 0.002 & 0.0038 & 0.53 \\
School and Affiliated Hospitals & & & 0.039 \\
in millions & & 0.009 & 0.53 \\
NIH Research Grant Funds Per & -0.02 & & 0.56 \\
Faculty Member in thousands & & 2.56 & \\
Residency Directors Assessment & 1.61 & & \\
Score out of 5 & & -1.50 & \\
Peer Assessment Score out of 5 & & &
\end{tabular}




\section{Fit Diagnostics for STEP1}

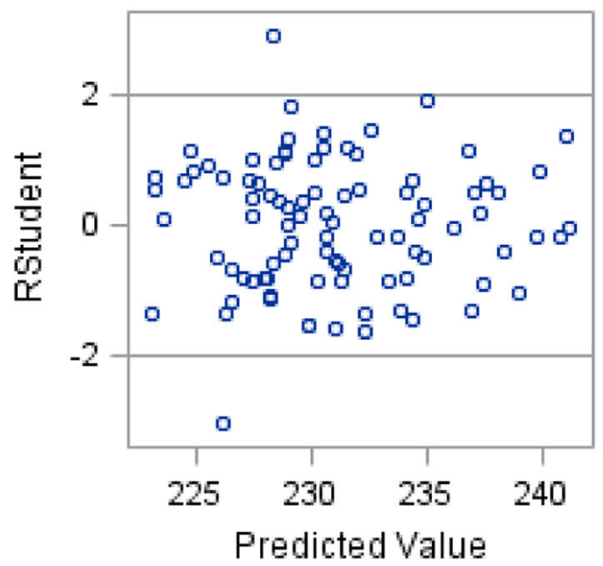

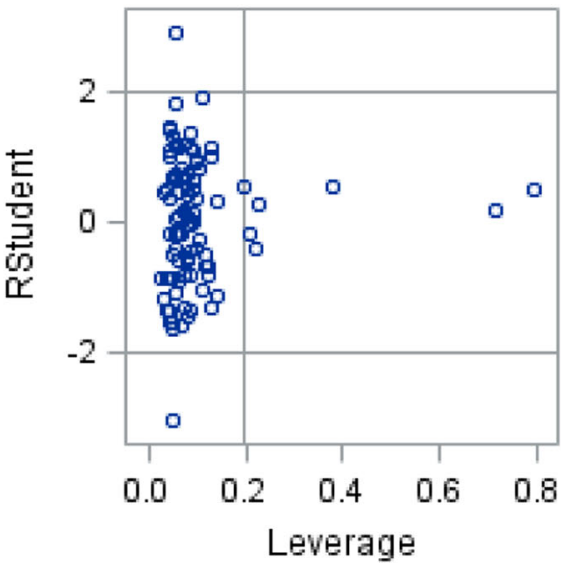

Fig. 1 Fit Diagnostics for STEP1

Therefore, states have an impetus to ensure that public medical schools are socially accountable by producing the much-needed primary care practitioners; hence accounting for public schools producing graduates who are more likely to choose primary care careers versus students trained in private medical schools [19]. With a greater likelihood of pursuing primary care, students in public institutions are less likely to pursue specialties which require more competitive Step scores, thus by extension yielding in public schools having slightly lower scores [20].

The only significant variable in the multivariable regression analysis model for Step 1 score is median MCAT score (Table 3), whereas NIH research grant funds per faculty member are an additional significant variable associated with Step 2 scores (Table 4). Surprisingly, the amount of grant funding schools received correlated inversely with Step 2 scores. There may be various explanations for this: perhaps the faculty at schools without abundant grant funding spend less time on research and more in patient care and teaching $[21,22]$. However, the correlation between NIH research grant funds and Step 2 scores may also be explained by the outliers and the schools with high lev values in our dataset, which may affect regression coefficient estimates. Hence, more research should be conducted regarding this association.

Fit Diagnostics for Step 1 and 2 reveal several potential outliers (Figs. 1 and 2). University of Missouri-Columbia consistently outperforms on Step 1 and 2, despite accepting medical students with lower MCAT scores than the national average [23]. One possible explanation for the outliers may be unique features of their curriculum. Of note, curricular (i.e. early clinical exposure, minimized lecture time, and focus on clinical vignettes in a "patient-based learning" style) as well as administrative

\section{Fit Diagnostics for STEP2}

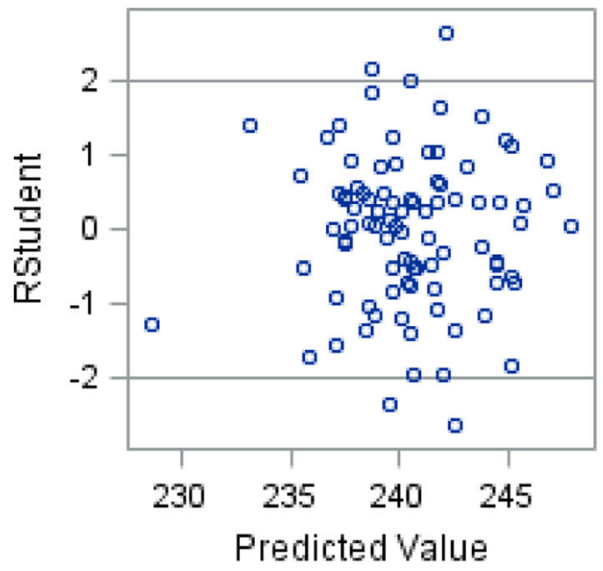

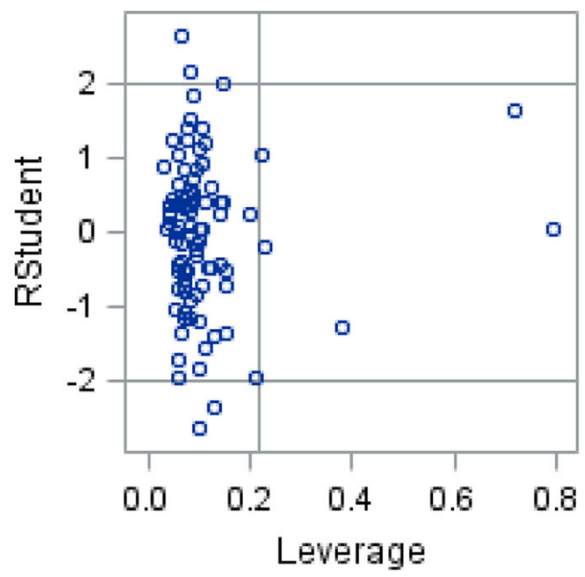

Fig. 2 Fit Diagnostics for STEP2 
changes in 1993 to improve University of Missouri-Columbia's medical curriculum, may have contributed to their success on the USMLE [23]. Furthermore, better exam performance may partially be explained by greater clinical exposure in the curriculum early on, where the first 2 years at the University of Missouri-Columbia are utilized for early clinical exposure and basic science education [23]. Overall, further evaluation of the curriculum at schools exceeding predictions of Step scores should be conducted to determine what is being done differently from other U.S. medical schools.

The fact the University of Missouri-Columbia is the only medical school in the United States to outperform in both Step 1 and Step 2 should draw special attention to determining what specifics of the curriculum and/or administrative organization contribute to their success. If these variables can be determined, they can be utilized at other institutions, and in turn enhance student learning. Another benefit of replicating the successes of the University of Missouri-Columbia would be that medical schools can minimize concern about board examination underperformance by students with lower than average MCAT scores, and instead place more emphasis on selecting students for admissions based on institution mission.

\section{Conclusions}

This study uncovers several medical schools which outperform or underperform trend line expectations for USMLE, irrespective of entering student qualifications. One outlier institution, the University of MissouriColumbia, was found to significantly outperform in both Step 1 and 2; such performance may be explained by curriculum and administrative differences. Having identified institutions that outperform expectations, the next sequence of investigations should aim to pinpoint the nuances within the "patient-based learning" curriculum that helped enhance medical education at the University of Missouri-Columbia. If these variables can be determined and disseminated, institutions globally will be able to produce physicians with greater clinical knowledge and skills, thereby improving patient care.

\section{Abbreviations \\ CK: Clinical Knowledge; GPA: Grade point average; MCAT: Medical College Admissions Test; NIH: National Institute of Health; USMLE: United States Medical Licensing Examination; USN\&WR: U.S. News and World Report}

\section{Acknowledgements}

Not applicable.

\section{Funding}

R.F. was partially supported by grants from NIH/NIMHD U54MD007584 for the analysis of data.

\section{Availability of data and materials}

Data was collected from a publicly accessible database, USN\&WR, and does not contain specific student identifiers. Only publicly available data was utilized in our analysis at the time of data collection. However, the data that support the findings of this study are available from USN\&WR but restrictions apply to the availability of these data, which were used under license for the current study, and so are not publicly available currently. Data are however available from the authors upon reasonable request and with permission of USN\&WR.

\section{Authors' contributions}

A.G., R.L., J.M., contributed to the conceptual framework, research question, critical revision and design of the study. A.G., R.L., contributed to the literature review, writing the manuscript, acquisition and interpretation of data for the work. R.F. contributed to the analysis of data for the work. All authors read and approved the final manuscript.

\section{Ethics approval and consent to participate}

Institutional review board exemption for waivers of informed consent was attained from the University of Hawai'i at Mānoa, Office of Research Compliance.

Consent for publication

Not applicable.

\section{Competing interests}

The authors declare that they have no competing interests.

\section{Publisher's Note}

Springer Nature remains neutral with regard to jurisdictional claims in published maps and institutional affiliations.

Received: 26 November 2018 Accepted: 13 May 2019

Published online: 20 May 2019

\section{References}

1. Kirch D, Prescott J. From rankings to Mission. Acad Med. 2013;88(8):1064-6.

2. Goldstein M, Lunn M, Peng L. What makes a top research medical school? A call for a new model to evaluate academic physicians and medical school performance. Acad Med. 2015;90(5):603-8.

3. Hendrix D. An analysis of bibliometric indicators, National Institutes of Health funding, and faculty size at Association of American Medical Colleges medical schools, 1997-2007. Journal of the Medical Library Association: JMLA. 2008;96(4):324-34.

4. Hu Y, Martindale J, LeGallo R, White C, McGahren E, Schroen A. Relationships between preclinical course grades and standardized exam performance. Adv Health Sci Educ. 2015;21(2):389-99.

5. Torre D, Papp K, Elnicki M, Durning S. Clerkship directors' practices with respect to preparing students for and using the National Board of medical examiners subject exam in medicine: results of a United States and Canadian survey. Acad Med. 2009;84(7):867-71.

6. Green $M$, Jones $P$, Thomas J. Selection criteria for residency: results of a National Program Directors Survey. Acad Med. 2009;84(3):362-7.

7. Basco W, Way D, Gilbert G, Hudson A. Undergraduate institutional MCAT scores as predictors of USMLE step 1 performance. Acad Med. 2002; 77(Supplement):S13-6.

8. Julian E. Validity of the medical college admission test for predicting medical school performance. Acad Med. 2005;80(10):910-7.

9. Hojat M, Erdmann J, Veloski J, Nasca T, Callahan C, Julian E, et al. A validity study of the writing sample section of the medical college admission test. Acad Med. 2000;75(Supplement):S25-7.

10. Donnon T, Paolucci E, Violato C. The predictive validity of the MCAT for medical school performance and medical board licensing examinations: a meta-analysis of the published research. Acad Med. 2007;82(1):100-6.

11. Wiley A, Koenig J. The validity of the medical college admission test for predicting performance in the first two years of medical school. Acad Med. 1996:71(10):S83-5.

12. Case S, Ripkey D, Swanson D. The relationship between clinical science performance in 20 medical schools and performance on step 2 of the USMLE licensing examination. 1994-95 validity study group for USMLE step 1 and 2 pass/fail standards. Acad Med. 1996;71(1):S31-3.

13. Ripkey D, Case S, Swanson D. Predicting performances on the NBME surgery subject test and USMLE step 2. Acad Med. 1997;72(10):S3-S33.

14. Ripkey D, Case S, Swanson D. Identifying students at risk for poor performance on the USMLE step 2. Acad Med. 1999;74(10):S45-8. 
15. Roth K, Riley W, Brandt R, Seibel H. Prediction of students' USMLE step 2 performances based on premedical credentials related to verbal skills. Acad Med. 1996;71(2):176-80.

16. USMLE Score Interpretation Guidelines* [Internet]. United States Medical Licensing Examination; 2017 [cited 18 June 2017]. Available from: http:// www.usmle.org/pdfs/transcripts/USMLE_Step_Examination_Score_ Interpretation_Guidelines.pdf

17. Facts Table 16 [Internet]. American Association of Medical Colleges; 2017 [cited 18 June 2017]. Available from: https://www.aamc.org/download/ 321494/data/factstablea16.pdf

18. Gauer J, Wolff J, Jackson J. Do MCAT scores predict USMLE scores? An analysis on 5 years of medical student data. Medical Education Online. 2016; 21(1):31795.

19. Washko M, Snyder J, Zangaro G. Where do physicians train? Investigating public and private institutional pipelines. Health Aff. 2015;34(5):852-6.

20. Charting Outcomes in the Match Characteristics of Applicants Who Matched to Their Preferred Specialty in the 2014 Main residency match [internet]. 5th ed. Washington D.C.: National Resident Matching Program; 2017 [cited 18 June 2017]. Available from: http://www.nrmp.org/wp-con tent/uploads/2014/09/Charting-Outcomes-2014-Final.pdf

21. Zinner D. Life-science research within US academic medical centers. JAMA. 2009;302(9):969

22. Meador K. Decline of clinical research in academic medical centers. Neurology. 2015;85(13):1171-6.

23. Blake R, Hosokawa M, Riley S. Student performances on step 1 and step 2 of the United States medical licensing examination following implementation of a problem-based learning curriculum. Acad Med. 2000;75(1):66-70.

Ready to submit your research? Choose BMC and benefit from:

- fast, convenient online submission

- thorough peer review by experienced researchers in your field

- rapid publication on acceptance

- support for research data, including large and complex data types

- gold Open Access which fosters wider collaboration and increased citations

- maximum visibility for your research: over $100 \mathrm{M}$ website views per year

At $\mathrm{BMC}$, research is always in progress.

Learn more biomedcentral.com/submissions 